\title{
Dermoscopy of Dermatomyositis in Dark Skin
}

\author{
Shekhar Neema ${ }^{1}$, Rohit Kothari ${ }^{1}$, Ahmed Waheed Kashif ${ }^{1}$, Deepak Vashisht ${ }^{1}$, \\ Biju Vasudevan ${ }^{1}$
}

1 Armed Forces Medical College, Pune, India

Key words: dermatomyositis, dermoscopy, dermatoscopy

Citation: Neema S, Kothari R, Kashif AW, Vashisht D, Vasudevan B. Dermoscopy of dermatomyositis in dark skin. Dermatol Pract Concept. 2022;12(1):e2022013. DOI: https://doi.org/10.5826/dpc.1201a13

Accepted: May 8, 2021; Published: January 2022

Copyright: $₫ 2022$ Neema et al. This is an open-access article distributed under the terms of the Creative Commons Attribution-NonCommercial License (BY-NC-4.0), https://creativecommons.org/licenses/by-nc/4.0/, which permits unrestricted noncommercial use, distribution, and reproduction in any medium, provided the original authors and source are credited.

Funding: None.

Competing interests: None.

Authorship: All authors have contributed significantly to this publication.

Corresponding author: Shekhar Neema MD, FEBDV, Associate Professor, Armed Forces Medical College, Pune, India.

E-mail: shekharadvait@gmail.com

\section{Introduction}

Dermatomyositis is an idiopathic inflammatory myopathy characterized by muscle weakness and cutaneous features such as heliotrope rash, Gottron papule, confluent macular erythema, poikiloderma, mechanic's hand, ragged cuticle and periungal telangiectasia [1]. The characteristic cutaneous manifestations are difficult to appreciate in individuals with darker skin types thus making the diagnosis challenging.

\section{Case Presentation}

A 59-year-old woman presented with complaints of pruritic dark lesions over her entire body and progressive muscle weakness of 2 months' duration. Examination revealed hyperpigmentation involving the face, periocular region (heliotrope rash), upper back (Shawl sign), and $\mathrm{v}$ area of neck (V sign), hyperpigmented papules over the knuckles (Gottron papule), hyperpigmented macules on the hand (Gottron sign), hyperpigmented papules over the lateral aspect of the index finger and medial aspect of thumb (mechanic's hand), and ragged cuticles (Figure 1, A-D).
Dermoscopy of the peri-ocular area revealed a brown reticular pigment network, gray dots and globules and linear out-of-focus vessels (Figure 1E). Dermoscopy of the Gottron papule shows white-to-pink structureless areas and a reticular pigment network (Figure 1F). Histopathology revealed atrophic epidermis, basal cell vacuolation, melanophages in the papillary dermis, and presence of focal mucin (Figure 1G). Creatinine phosphokinase was $573 \mathrm{IU} / \mathrm{ml}$, antinuclear antibody was $3+$ (speckled pattern). Magnetic resonance imaging of the thigh showed myositis, and high-resolution computed tomography of chest were suggestive of interstitial lung disease. A diagnosis of dermatomyositis was made based on clinical, histopathological, serological, and imaging findings. The patient was treated with methylprednisolone pulse of $1 \mathrm{~g}$ per day for 3 days, hydroxychloroquine $200 \mathrm{mg}$ once a day and a tapering dose of oral steroids.

In a second case, a 45-year-old woman presented with similar complaints of photosensitivity, pruritic dark lesions over photo-exposed areas of the face, trunk, and extremities, swelling around the eyes, and proximal muscle weakness. Dermatological examination showed hyperpigmented macules over the forehead and lateral sides of cheeks and 


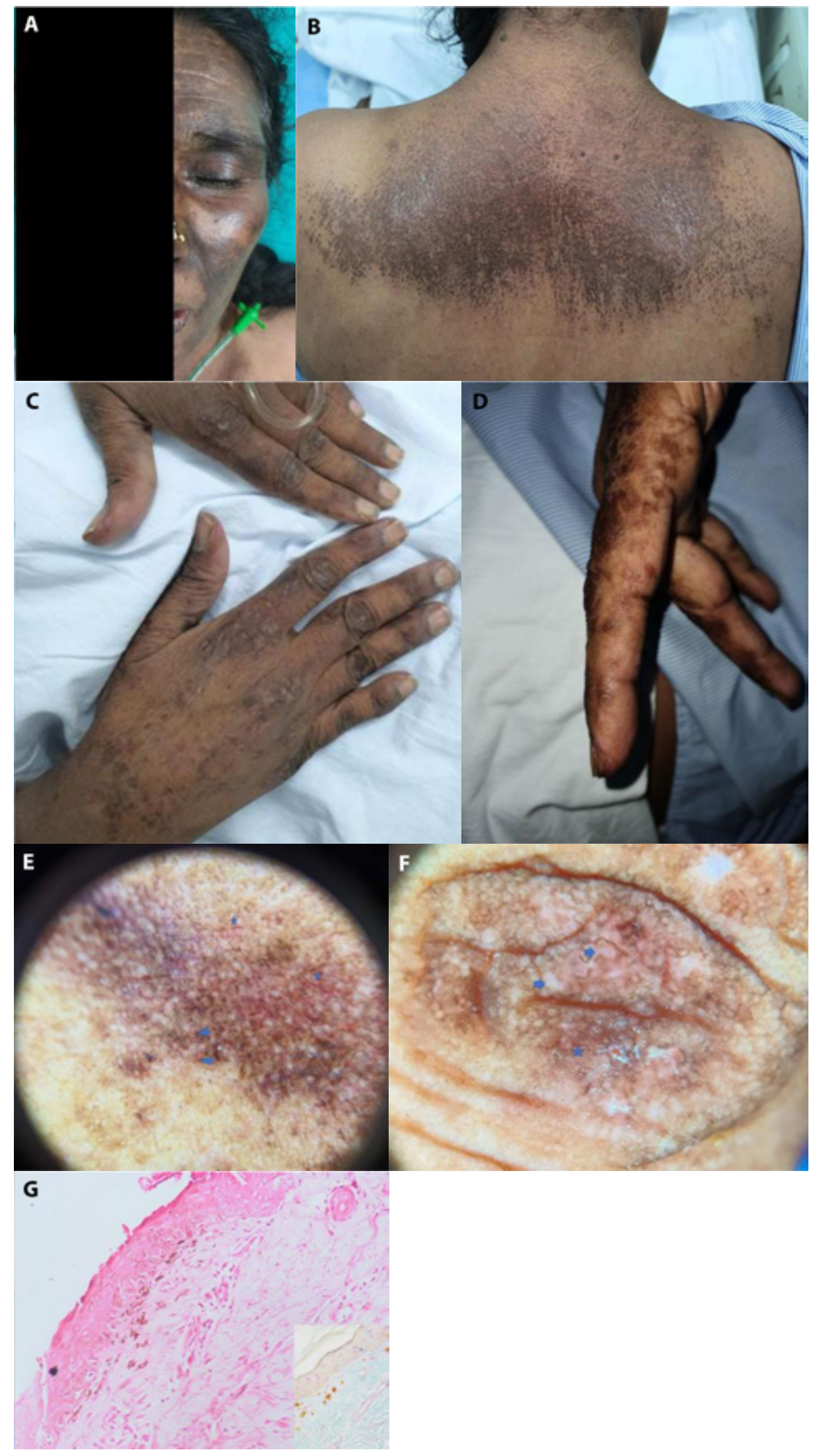

Figure 1. (A) Diffuse hyperpigmentation of face including peri-ocular (heliotrope rash), zygomatic, and nasolabial folds. (B) Hyperpigmented plaque over upper back (Shawl sign). (C) Hyperpigmented papules and macules over the knuckles (Gottron papule and Gottron sign). (D) Hyperpigmented papules over radial aspect of index finger (mechanic's hand). (E) Dermoscopy of heliotrope rash shows brown reticular network, gray dots and globules (blue arrows) and linear out-of-focus vessels (blue stars) (DermLite DL4, polarized, $\times 10$ ). (F) Dermoscopy of Gottron papule shows white-to-pink structureless areas (blue arrows) and a reticular pigment network (blue star) (DermLite DL4, polarized, $\times 10)$. (G) Histopathological examination shows an atrophic epidermis, basal cell vacuolation, melanophages, perivascular lymphocytic infiltrate and the presence of focal mucin (hematoxylin and eosin, $\times 200$ ). PAS stain shows the presence of mucin (inset). 


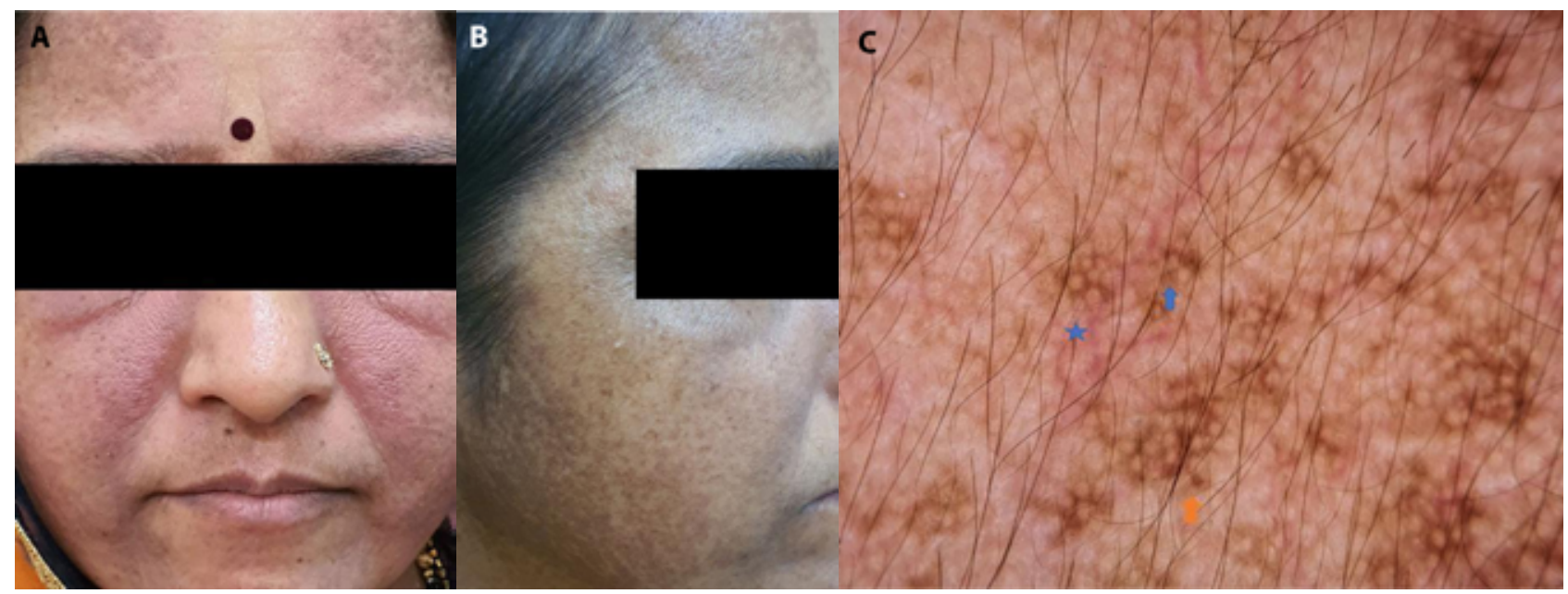

Figure 2. (A) Hyperpigmented macules over the forehead and erythema and edema over the malar area. (B) Hyperpigmented macules over the lateral aspect of the cheek. (C) Dermoscopy shows brown reticular network (orange arrow), gray dots and globules (blue arrow), and out-of-focus linear vessels (blue star) (DermLite DL4, polarized, $\times 10$ ).

erythema and edema involving the malar area (Figure 2, A and B). Dermoscopy showed a reticular pigment network, brownto-gray dots and globules, and linear vessels (Figure 2D). The imaging, biochemical investigations, histopathology, and serology was consistent with diagnosis of dermatomyositis.

\section{Conclusion}

Cutaneous features are essential and characteristic in the diagnosis of dermatomyositis. Dermoscopy of Gottron papule has been described as a lump of surface scales and dotted vessels [2]. Individuals with darker skin present with predominant hyperpigmentation, that poses a diagnostic difficulty. Pigment incontinence that is seen in histopathology is due to basal cell damage and is seen as dots and globules on dermoscopy. Linear out-of-focus vessels are due to epidermal atrophy, and focal mucin deposition is seen as structureless white areas on dermoscopy. A reticular pattern on dermoscopy occurs due to melanization of the basal layer.
Dermoscopy may be helpful in the diagnosis of cutaneous features of dermatomyositis in darker skin. We also tried to correlate dermoscopic findings with histopathology. This is the first report on dermoscopy of dermatomyositis in darker skin to the best of our knowledge.

Informed consent: Written informed consent for publication of her clinical details and clinical images was obtained from the patient.

\section{References}

1. Findlay AR, Goyal NA, Mozaffar T. An overview of polymyositis and dermatomyositis. Muscle Nerve. 2015;51(5):638-656. DOI:10.1002/mus.24566. PMID: 25641317.

2. Namiki T, Hashimoto T, Hanafusa T, Miura K, Yokozeki H. Case of dermatomyositis with Gottron papules and mechanic's hand: dermoscopic features. J Dermatol. 2018;45(1):e19-e20. DOI: 10.1111/1346-8138.14072. PMID: 28971518. 